\title{
Temporal and Spatial Foraging Behavior of the Larvae of the Fall Webworm Hyphantria cunea
}

\author{
Terrence D. Fitzgerald \\ Department of Biological Sciences, State University College at Cortland, Cortland, NY 13045, USA \\ Correspondence should be addressed to Terrence D. Fitzgerald; fitzgerald@cortland.edu
}

Received 14 July 2015; Accepted 24 November 2015

Academic Editor: Jacques Hubert Charles Delabie

Copyright (C) 2015 Terrence D. Fitzgerald. This is an open access article distributed under the Creative Commons Attribution License, which permits unrestricted use, distribution, and reproduction in any medium, provided the original work is properly cited.

\begin{abstract}
During their first three larval stadia, caterpillars of Hyphantria cunea (Lepidoptera: Arctiidae) are patch-restricted foragers, confining their activity to a web-nest they construct in the branches of the host tree. Activity recordings of eight field colonies made over 46 colony-days showed that the later instars become central place foragers, leaving their nests at dusk to feed at distant sites and then returning to their nests in the morning. Colonies maintained in the laboratory showed that same pattern of foraging. In Ychoice laboratory experiments, caterpillars were slow to abandon old, exhausted feeding sites in favor of new food finds. An average of approximately $40 \%$ of the caterpillars in five colonies still selected pathways leading to exhausted sites at the onset of foraging bouts over those leading to new sites after feeding exclusively at the new sites on each of the previous four days. On returning to their nests in the morning, approximately $23 \%$ of the caterpillars erred by selecting pathways that led them away from the nest rather than toward it and showed no improvement over the course of the study. The results of these Y-choice studies indicate that, compared to other previously studied species of social caterpillars, the webworm employs a relatively simple system of collective foraging.
\end{abstract}

\section{Introduction}

While sibling aggregates of caterpillars are simple societies lacking the complexity of interaction that characterizes the classically eusocial insects, studies show that the various species exhibit a rich diversity of cooperative interactions. Indeed, collectively, social caterpillars exhibit a greater range of social behaviors than are found among those species traditionally classified as subsocial forms and eclipse those of species of thrips, beetles, and aphids recently added to the catalog of eusocial insects $[1,2]$. Their social repertoires include communal and cooperative foraging, trail based communication and recruitment, cooperative shelter building, collective thermoregulation, and antipredator defense [2].

Much of the interest in social caterpillars has focused on their collective foraging behavior. The most common mode of feeding found among social caterpillars involves patchrestricted foraging $[2,3]$. Species exhibiting this mode of foraging envelop patches of leaves with silk and feed within the web-nest. As they grow, they may enlarge their feeding arenas to include a series of more or less contiguous patches often linked by silk covered branch pathways. One of the most widely distributed and conspicuous patch-restricted foragers is the fall webworm, Hyphantria cunea (Lepidoptera: Arctiidae). In the northeastern USA, the moths appear in the late spring and oviposit several hundred eggs on the undersides of the leaves of host plants [4]. The caterpillars eclose from their eggs in late June and immediately initiate the construction of a web-nest which they inhabit for all of their larval life. As the caterpillars grow, they continually extend the boundaries of the nest to enclose new leaves on which they feed.

Berger [5] and Snodgrass [6] were the first to publish observations of the temporospatial foraging behavior of the fall webworm. While both agreed that the caterpillars are initially patch-restricted foragers, Berger [5] stated that, during the later stadia, caterpillars in colonies he observed travelled to distant feeding sites outside the nest to feed at night, thus behaving as central place foragers. Snodgrass [6], however, questioned these observations, stating that the colonies he observed fed solely within the nest throughout 


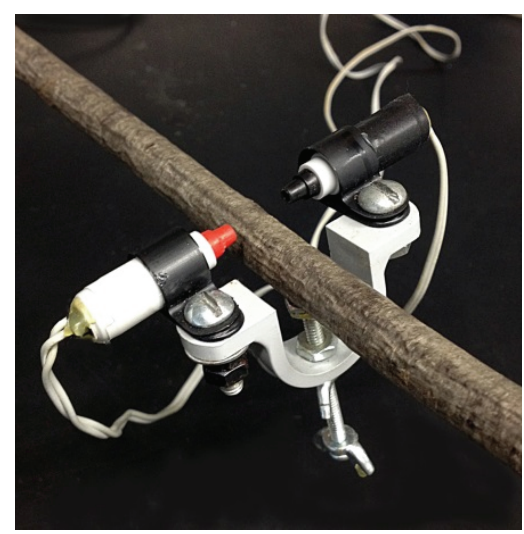

FIGURE 1: Infrared activity monitor.

their larval life. The distinction is significant because central place foraging sets the stage for the evolution of more advanced forms of cooperative foraging than occur among those species that are strictly patch-restricted foragers [3]. While other investigators have since reported on the temporal and spatial pattern of the foraging behavior of the webworm [7-9] neither these studies nor those of Berger [5] and Snodgrass [6] provide hard documentation of the temporal and spatial pattern of foraging of undisturbed field colonies. The present study was undertaken to provide that data set. The efficiency with which the colonies abandon exhausted feeding sites in favor of new food finds and the ability of caterpillars to find the way back to the nest from distant feeding sites were also investigated.

\section{Materials and Methods}

2.1. Source of Caterpillars. Studies of the temporal and spatial pattern of foraging of field colonies were conducted in 1997 on caterpillars collected from Cortland County, NY. Laboratory studies to determine the efficiency with which caterpillars abandoned exhausted feeding sites and their ability to return directly to the nest after feeding were conducted in 2014 on caterpillars collected from Lycoming County, PA.

2.2. Temporal and Spatial Pattern of Foraging. In addition to direct observations of field colonies made throughout their development, electronic recording of eight field colonies was undertaken from August 20 to September 21, 1997, to obtain information on the daily pattern of foraging of maturing caterpillars. To record colony activity, an infrared activity monitor was attached to the main branch-pathway extending from the nest in such a manner that caterpillars moving between their nests and feeding sites broke the infrared beam and triggered the detector ([10], Figure 1). Fall webworms spin copious quantities of silk and it was necessary in some instances to remove silk from the monitor at the onset of activity periods to assure that the caterpillars passed between the detector's emitter and sensor. Detector response was registered on a Hobo event logger (Onset Computer Co., Bourne, MA, http://www.onsetcomp.com/) and the data later uploaded to a computer and compiled with BoxCar software
(Onset Computer Co.). Activity over a total of 46 colony-days was recorded.

2.3. Efficiency of Abandoning Exhausted Feeding Sites. Ychoice experiments were conducted that required caterpillars to choose between alternate pathways, one of which led to an old, exhausted feeding site and the other to a new site. The nests of fifth to sixth instar caterpillars used in these studies were collected in the field and established on separate wooden stands made of $1 / 4$ inch dowels to resemble the branching pattern of a tree [11]. Colonies readily adapted to the stands, gradually expanding their nests over the expanse of the structures. Caterpillars in each colony were provided with a fully foliated branch of black cherry (Prunus serotina) as a food source which was abutted to the end of an elongated horizontal arm of the stand (the bridge). The branches were sufficiently large to provide the caterpillars with leaves in excess of their needs. The colonies were maintained in a room with windows admitting daylight so that the caterpillars were exposed to the same light regime as in the field. Experiments were initiated after the caterpillars had been established on the stands for at least one week. To conduct a Y-choice experiment, two additional sections of dowels, each $20 \mathrm{~cm}$ in length and previously unused, were arranged to extend the bridge leading to food. The proximal section of the extension was placed in line with the bridge while the distal section was abutted to the end of the proximal section at an angle of 15 degrees and the cherry branch placed at its end. Caterpillars were allowed to move over these sections and to feed ad libitum for the next two days. On the third day, another $20 \mathrm{~cm}$ long section of unused dowel was abutted to the end of the proximal section of the extension at an angle of 15 degrees. Thus the proximal stem section and the two distal sections (arms) were arranged in the form of a Y, separated by an angle of approximately $30^{\circ}$. Leaves remaining on the branch that the caterpillars had been feeding on the previous two days, located at the end of the first arm, were removed by hand but the branch was left in place and it and the arm leading to it were otherwise undisturbed. A new, fully foliated branch was placed at the end of the new arm. The number of caterpillars selecting each of the alternative arms of the $\mathrm{Y}$ as they moved from their nests to feed was determined by analyzing video recorded at the rate of one frame per second during each of the following five days. Thus, the study was designed to determine how long it would take the caterpillars to abandon the arm that led to the exhausted feeding site in favor of the arm that led to a new food supply. Night-time recording was facilitated by illuminating the $\mathrm{Y}$ with a $25 \mathrm{w}$ red light bulb. The study was replicated with five different colonies of fifth to sixth instar caterpillars.

2.4. Ability of the Caterpillars to Return Directly to the Nest after Feeding. As caterpillars return to their nest after feeding they must select between alternate pathways at branch junctures. The extent to which caterpillars select pathways that provided the most direct route back to the nest was observed under laboratory conditions. Caterpillars in each of the five laboratory colonies, established as described above, were monitored by camera as they returned to their nests after 

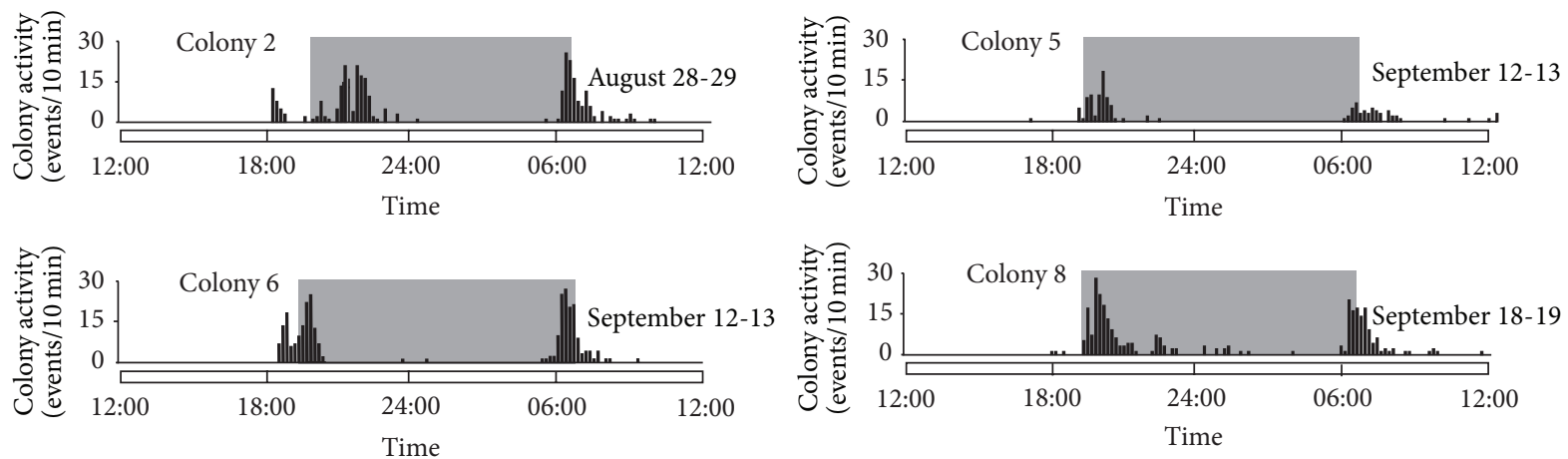

FIgURE 2: Activity records of field colonies of Hyphantria cunea caterpillars recorded in 1997. Grey rectangles indicate periods of darkness.

TABLE 1: Activity median (minutes \pm SE) after sunset (evening) and before sunrise (morning) for eight colonies of Hyphantria cunea.

\begin{tabular}{lccc}
\hline \multirow{2}{*}{ Colony } & \multirow{2}{*}{ Days recorded } & \multicolumn{2}{c}{ Activity median } \\
& & Evening & Morning \\
\hline 1 & 5 & $62.8 \pm 7.6$ & $38.0 \pm 6.9$ \\
2 & 7 & $67.7 \pm 12.6$ & $7.3 \pm 4.6$ \\
3 & 5 & $54.2 \pm 16.6$ & $6.0 \pm 9.0$ \\
4 & 3 & $87.6 \pm 9.6$ & $36.0 \pm 7.5$ \\
5 & 8 & $34.7 \pm 4.5$ & $1.3 \pm 7.8$ \\
6 & 8 & $51.4 \pm 7.6$ & $1.0 \pm 7.1$ \\
7 & 8 & $78.4 \pm 13.2$ & $20.7 \pm 8.6$ \\
8 & 2 & $57.5 \pm 4.5$ & $0.5 \pm 5.5$ \\
All colonies & 46 & $59.7 \pm 4.4$ & $12.7 \pm 3.1$ \\
\hline
\end{tabular}

feeding overnight. When a caterpillar reached the juncture of the stem and the alternate arm of the Y, it had to select between the two: the stem leading to the nest or the alternate arm leading away from the nest. Caterpillars choosing the alternate arm and moving along it to the old, exhausted feeding site were considered to make a mistake as this choice eventually required them to turn around to find their way back.

2.5. Statistical Analysis. Data were analyzed with SigmaStat statistical software. Proportions were subjected to arcsin sqrt transformation before analyses.

\section{Results}

3.1. Temporal and Spatial Pattern of Foraging. Direct observations of field colonies indicate that through the third stadium the caterpillars forage within the nest which is continually expanded to enclose new leaves. Recordings show that, in the subsequent stadia, the caterpillars are central place foragers, leaving the nest at dusk and traveling to distant feeding sites. Activity records of four colonies, typical of the temporal pattern of behavior exhibited by all eight of the field colonies, are shown in Figure 2. The activity median of caterpillars exiting the nest occurred approximately one hour after sunset (Table 1). Caterpillars in field colonies fed overnight then returned to their nests in the morning with the activity median of returning occurring approximately 13 minutes before sunrise. Although the majority of the caterpillars were back in the nest by $0800 \mathrm{~h}$, stragglers sometimes did not return until later in the morning (Figure 2). Caterpillars in all of our laboratory colonies maintained under an ambient daylight regime showed a similar pattern of nocturnal foraging (Figure 3).

\subsection{Efficiency of Abandoning Exhausted Feeding Sites. During} the first foray after the food supply was switched from the old to the new site, caterpillars initially selected the arm of the Y that led to the old site. After exploring the defoliated branch the caterpillars returned to the nest and then started out again, often several times. Some of the caterpillars eventually ventured onto the new arm of the $\mathrm{Y}$ but initially turned back after moving part way along its length. As they moved onto the new arm the caterpillars laid down strands of silk and the pathway became increasingly covered with the material. Successive waves of caterpillars to move onto the new arm of the Y showed decreased hesitancy and a lessened tendency to turn back. Trail marking in the FWW has not yet been investigated so it is not known if the physical/chemical properties of silk elicit trail following or if trail marking involves a pheromone secreted from some other site as has been shown for other caterpillars [2]. The time elapsed between the onset of the first foray after the food was switched and the arrival of the first caterpillar at the new food source was highly variable among colonies. The median interval was 27 minutes. Thereafter, caterpillars in increasing numbers selected the arm of the Y leading to the new food source and eventually all of the colony moved to the new site and fed. Of the total number of choices between $\mathrm{Y}$ arms made during the first foray after the feeding site was moved, $33.1 \pm 6.3 \%$ were for the new site. On the following evening $44.7 \pm 6.9 \%$ were for the new site and on the third evening $58.9 \pm 5.4 \%$ were for the new site (Figure 4). In all cases, all caterpillars in each colony eventually found their way to the new feeding site. Although the proportion of choices for the new site increased between the first and third days, it did not increase on the two subsequent days on which there was a marked variation in response among colonies (Figure 4). For the overall data set, there was no significant difference in the number of choices for the new site over time (ANOVA, $F=1.75, p=0.18$ ). 

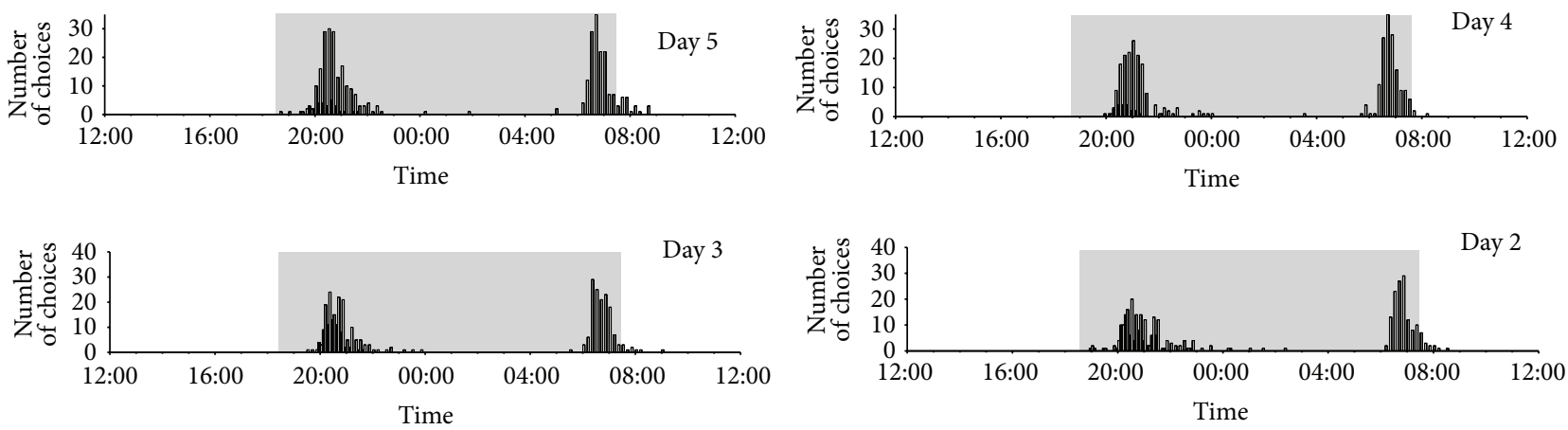

Day 1

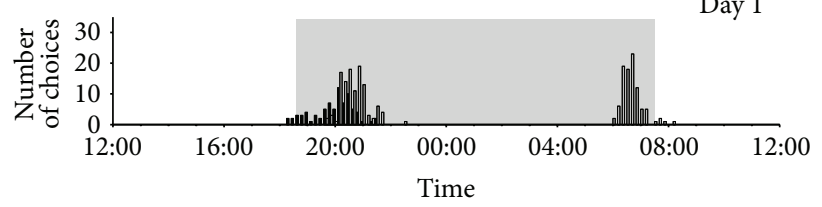

FIGURE 3: Activity patterns of caterpillars in a laboratory colony recorded over five consecutive days. The $x$-axis records the number of choices over five-minute intervals for the old site (dark bars) and the new site (hollow bars). Scotophase indicated by shaded area.

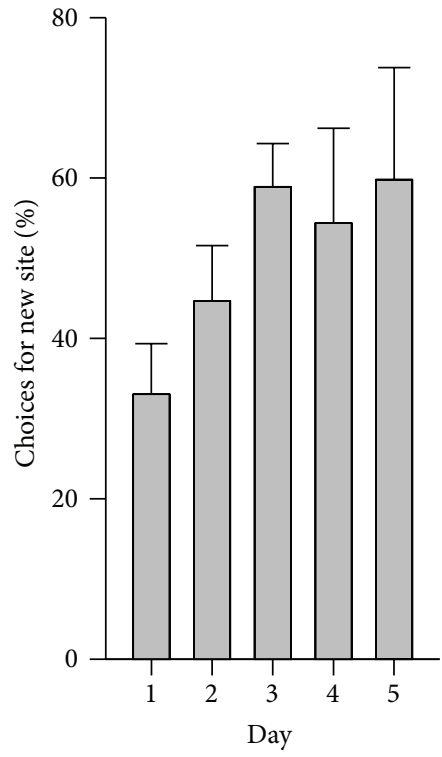

FIGURE 4: Mean percent $( \pm$ SE) choices by fall webworm caterpillars for arms of Ys leading to new feeding sites at onset of evening foraging bouts.

3.3. Ability of the Caterpillars to Return Directly to the Nest after Feeding. On returning to the nest on the first morning after the food supply was switched from the old to the new site $22.5 \pm 8.8 \%$ of the caterpillars in the five colonies selected and moved onto the arm of the $\mathrm{Y}$ that led to the old feeding site rather than the stem of the $\mathrm{Y}$ that led directly to the nest. On the second and third days of the experiment, $21.2 \pm 7.8 \%$ and $25.4 \pm 7.1 \%$ of caterpillars, respectively, initially chose the arm that led to the old feeding site over the stem. There was no difference among responses over the three days (ANOVA, $F=0.09, p=0.92$ ).

\section{Discussion}

4.1. Temporal and Spatial Pattern of Foraging. In this study, late instar caterpillars adopted a central place foraging mode consistent with the observations first reported by Berger [5]. Although Snodgrass [6] disagreed with Berger on this point, stating that the colonies he observed foraged only in the proximity of the nest, subsequent studies suggest that Snodgrass observed a population of the caterpillar that behaved differently than the caterpillars observed by Berger. There are two color morphs of the FWW and Oliver [7] noted that while the larvae of both morphs he studied in Arkansas fed in contact with the nest during the early instars, during the last three stadia, caterpillars of what he termed the "orange race" left the nest at night to feed and then returned in the morning, whereas those of the "black race" did not. Ito and Warren [8] and Takeda [9] also reported that the behavior of the two color types differed in a manner consistent with that first noted by Oliver [7] though they referred to the lighter colored caterpillars as a "red headed type." There is no reproductive isolation between the color forms and Morris [12] and Hattori and Ito [13] observed colonies with "mosaic" head colors that behaved like the red headed type. A distribution map drawn by Ito and Hattori [14] shows the black headed form occurring in a region that includes Connecticut where Snodgrass [6] made his observations and a mosaic form occurring in Ohio where Berger [5] made his. Indeed, Snodgrass described his caterpillars as having black heads. Berger, however, reported considerable variation in the head colors of the caterpillars in the colonies he studied, ranging from black to light brown, but did not record any differences in the temporal or spatial foraging patterns of the colonies; all adopted the central place mode of foraging.

Although Ito and Hattori [14] concluded that the red headed type was restricted to the southern United States, both the red and black headed forms were common in Cortland 
County, New York, in 1997 when the first phase of the study reported here was conducted. Of 18 colonies observed in the study area, seven consisted of purely black headed caterpillars, two of purely red headed caterpillars, and eight of a mixture of both black headed and red headed caterpillars. In 2014, colonies were collected from near Williamsport, Pennsylvania, as no local colonies could be found. All of 20 colonies that were collected in Pennsylvania consisted of caterpillars with the mosaic head color. As reported here, all caterpillars in the eight colonies recorded in 1997 and the five colonies in 2014 foraged only at night at sites distant from the nest and then returned in the morning. The colonies whose behavior was recorded in 1997 were of mixed black and red headed types with the exception of colony 4 which was a colony of the pure black headed type. As shown in Table 1, colony 4 exhibited the same pattern of nocturnal foraging as the mixed colonies. The results of this and the previous studies noted above indicate that there is considerable temporal and spatial heterogeneity in the relationship between color morphism and larval foraging patterns in the webworm.

4.2. Ability of the Caterpillars to Return Directly to the Nest after Feeding. Caterpillars observed in field colonies during the present study broke into small groups or fed alone when they left the nest in the evening and became widely dispersed, yet all the caterpillars eventually returned to the nest each morning. Observations made while caterpillars were returning to their nests following these overnight forays both in the laboratory and in the field indicate that the webworm extracts less information from its pathways than previously studied species of central place foragers [2]. Studies of these other species showed that they are able to distinguish between pathways at branch junctures that differ in strength and age and that this allows caterpillars to move efficiently from the feeding site back to the nest. Unlike these other species, between 22 and $25 \%$ of the choices made by webworms in our laboratory colonies at the $\mathrm{Y}$ path juncture on three consecutive mornings as they returned to the nest after feeding overnight were for pathways that led away from the nest rather than toward it. Caterpillars returning to their nests following their overnight feeding bouts in the field were also observed to make mistakes at branch junctures that took them farther away from their nests. During one set of observations, each of five-minute duration, made as caterpillars were returning to their nests in the morning, an average of $9.7 \pm 2.2$ caterpillars ( $n=3$ colonies) were observed to make choice errors that resulted in them moving away from rather than toward their nests. In addition, as shown in Figure 2, a small percentage of caterpillars in colonies typically returned to their nests much later in the morning than the majority suggesting that these individuals could have had particular difficulty finding their way back.

4.3. Efficiency of Abandoning Exhausted Feeding Sites. Although there have been no studies of chemical communication in the larval webworm, the results of the study presented here indicate that, in contrast to other social caterpillars that are central place foragers, the webworm employs a relatively simple system of communication that may do a little more than facilitate aggregation and collective nest construction. Compared to the eastern tent caterpillar, an ecological equivalent of the webworm which feeds on $P$. serotina in the spring and is a central place forager, the present study shows that the webworm is relatively inefficient not only in finding its way back to its nest but also in abandoning exhausted feeding sites in favor of new food finds. When tested under similar conditions using the same experimental protocol, colonies of the tent caterpillar abandoned exhausted feeding sites on the same evenings that they discovered and fed at new sites and did not return to the old sites on subsequent evenings [15]. In contrast, a significant proportion of the caterpillars in our laboratory colonies of webworms continued to return to exhausted feeding sites even after feeding for the previous four days at new food finds. This rapid shift to a new feeding site by the tent caterpillar is attributable to the ability of the insect to recruit nestmates to new food finds with a trail pheromone [15]. The central place foragers Eucheria socialis [16], Gloveria sp. [11], and Eutachyptera psidii [17] tested in the same manner showed that while they are less efficient than the tent caterpillar in abandoning depleted sites, they performed markedly better than the webworm with fewer than $20 \%$ of the caterpillars returning to the old site on the second night following the shift in the food source. In contrast, a similar study of the pine processionary, Thaumetopoea pityocampa [15], showed that the caterpillars of this species are slow to move to a new feeding site with approximately the same percentage of caterpillars as the webworm choosing the arm that led to the old depleted feeding site on the third day of the study.

Although fall webworms may show some preference for leaves based on age and extent of shading [18], they are relatively nonselective in their choice of food compared to $M$. americanum, the larvae of which strongly prefer the youngest leaves, which occur at the tips of branches [19]. In the spring when tent caterpillars are feeding on P. serotina there is high heterogeneity for leaf age as the trees continuously produce new leaves. The same host trees have largely stopped producing new leaves when the larvae of the webworm eclose and for the most part the caterpillars feed on aged leaves throughout their larval life that are tough and dry, for which little or no searching is required. The difference in the amount of information that the webworm and the tent caterpillar encode in and extract from their pathways likely reflects this difference in their response to leaf quality and the patchiness of the food resource.

\section{Conflict of Interests}

The author declares that there is no conflict of interests regarding the publication of this paper.

\section{Acknowledgment}

Katelyn Meyer assisted in the analysis of data collected during the laboratory component of the study. 


\section{References}

[1] J. T. Costa and T. D. Fitzgerald, "Social terminology revisited: where are we ten years later?” Annales Zoologici Fennici, vol. 42, no. 6, pp. 559-564, 2005.

[2] J. T. Costa, The Other Insect Societies, Belknap/Harvard University Press, Cambridge, Mass, USA, 2006.

[3] J. T. Costa and N. E. Pierce, "Social evolution in the Lepidoptera: ecological context and communication in larval societies," in Social Competition and Cooperation in Insects and Arachnids, Vol. II: Evolution of Sociality, J. C. Choe and B. J. Crespi, Eds., pp. 407-442, Cambridge University Press, Cambridge, Mass, USA, 1997.

[4] W. T. Johnson and H. H. Lyon, Insects that Feed on Trees and Shrubs, Cornell University Press, Ithaca, NY, USA, 1988.

[5] E. W. Berger, "Observations upon the migrating, feeding, and nesting habits of the fall webworm (Hyphantria cunea Dru.)," Bureau of Entomolology, USDA Bulletin, vol. 60, pp. 41-51, 1906.

[6] R. E. Snodgrass, “The fall webworm," Annual Report, Smithsonian Institution, Washington, DC, USA, 1923.

[7] A. D. Oliver, "A behavioral study of two races of the fall webworm, Hyphantria cunea, (Lepidoptera: Arctiidae) in Louisiana," Annals of the Entomological Society of America, vol. 57, no. 2, pp. 192-194, 1964.

[8] Y. Ito and L. O. Warren, "Status of black-headed and red-headed types of Hyphantria cunea (Drury) (Lepidoptera: Arctiidae). I biology of two types and results of crossing experiment," Applied Entomology and Zoology, vol. 8, pp. 157-171, 1973.

[9] M. Takeda, "Differentiation in life cycle of sympatric populations of two forms of Hyphantria moth in central Missouri," Entomological Science, vol. 8, no. 2, pp. 211-218, 2005.

[10] T. D. Fitzgerald and D. L. A. Underwood, "Winter foraging patterns and voluntary hypothermia in the social caterpillar Eucheria socialis," Ecological Entomology, vol. 25, no. 1, pp. 3544, 2000.

[11] T. D. Fitzgerald and D. L. A. Underwood, "Communal foraging behavior and recruitment communication in Gloveria sp.," Journal of Chemical Ecology, vol. 24, no. 8, pp. 1381-1396, 1998.

[12] R. F. Morris, "Notes on synonymy and color variation in the fall webworm Hyphantria cunea Drury," The Canadian Entomologist, vol. 95, no. 11, pp. 1217-1223, 1963.

[13] I. Hattori and Y. Ito, "Status of black-headed and red-headed types of Hyphantria cunea (Drury) (Lepidoptera: Arctiidae). II. External characters of the two types and their hybrids," Applied Entomology and Zoology, vol. 8, no. 3, pp. 172-182, 1973.

[14] Y. Ito and I. Hattori, "Status of black-headed and red-headed types of Hyphantria cunea (Drury) (Lepidoptera: Arctiidae). III. Distribution of various types and the discussion of the relationship among them," Applied Entomology and Zoology, vol. 10, no. 3, pp. 189-202, 1975.

[15] T. D. Fitzgerald, "Role of trail pheromone in foraging and processionary behavior of pine processionary caterpillars Thaumetopoea pityocampa," Journal of Chemical Ecology, vol. 29, no. 3, pp. 513-532, 2003.

[16] T. D. Fitzgerald and D. L. A. Underwood, "Trail marking by the larva of the madrone butterfly Eucheira socialis and the role of the trail pheromone in communal foraging behavior," Journal of Insect Behavior, vol. 11, no. 2, pp. 247-263, 1998.

[17] T. D. Fitzgerald, A. Pescador-Rubio, and G. Isaacs, "Foraging behaviour of the social caterpillar Eutachyptera psidii (Sallé) (Lepidoptera: Lasiocampidae) during a prolonged period of food and water deprivation," Ecological Entomology, vol. 33, no. 6, pp. 727-734, 2008.

[18] P. Barbosa and J. A. Greenblatt, "Effects of leaf age and position on larval preferences of the fall webworm Hyphantria cunea Drury," The Canadian Entomologist, vol. 111, pp. 381-383, 1978.

[19] T. D. Fitzgerald, The Tent Caterpillars, Cornell University Press, Ithaca, NY, USA, 1995. 

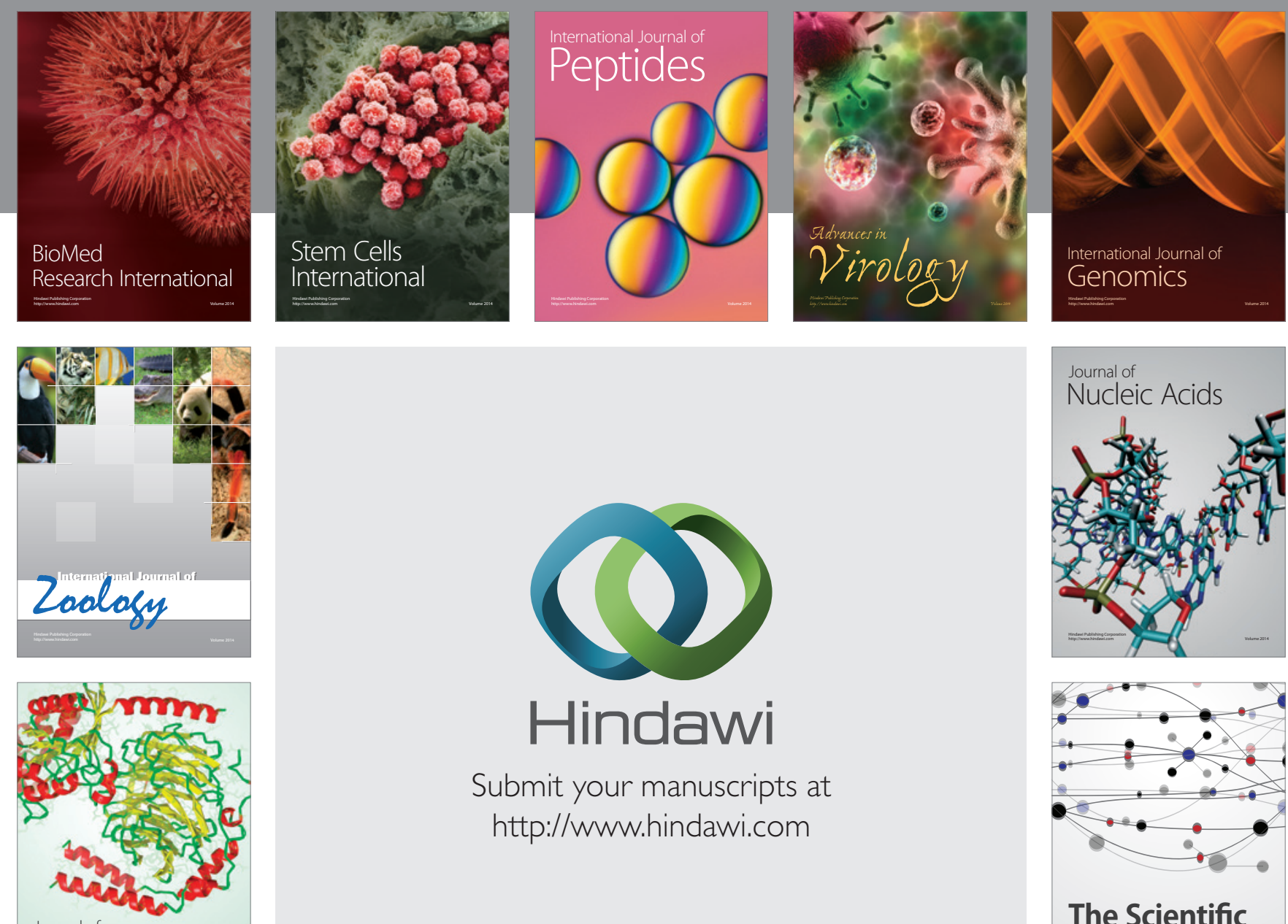

Submit your manuscripts at

http://www.hindawi.com

Journal of
Signal Transduction
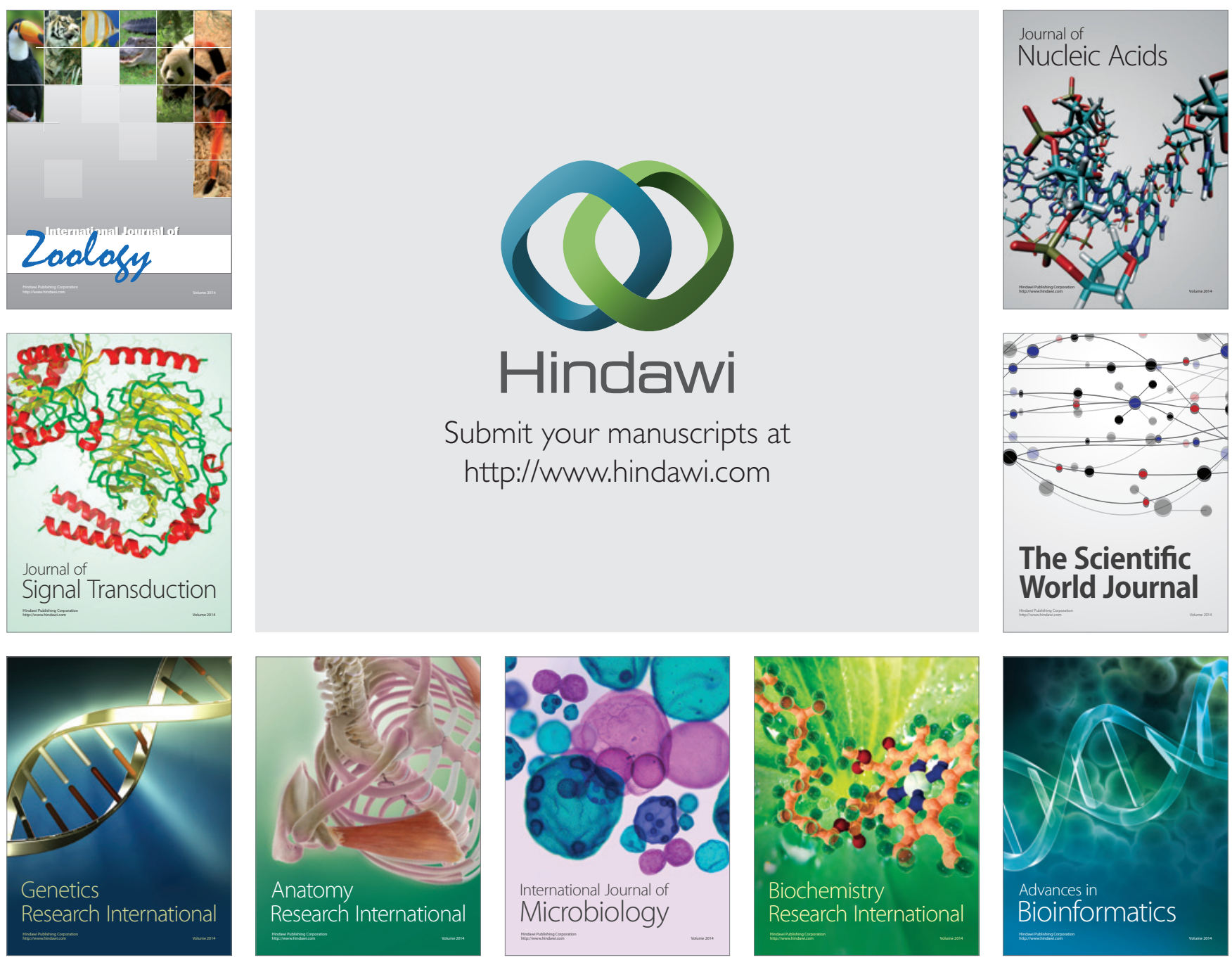

The Scientific World Journal
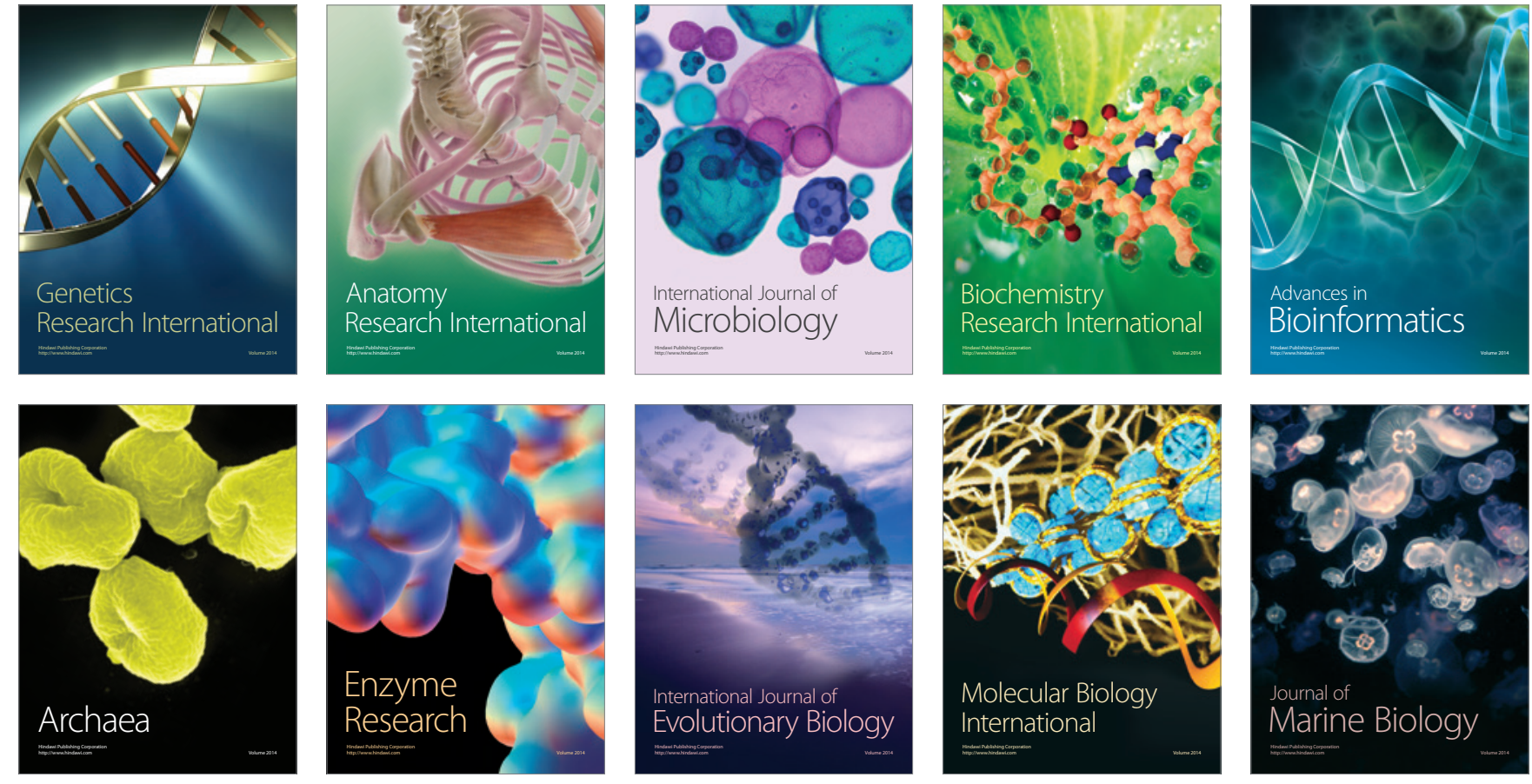